\title{
Bringing Everyday Applications to Interactive Surfaces
}

\author{
Malte Weiss \\ RWTH Aachen University \\ 52064 Aachen, Germany \\ weiss@cs.rwth-aachen.de
}

\begin{abstract}
This paper presents ongoing work that intends to simplify the introduction of everyday applications to interactive tabletops. SLAP Widgets bring tangible general-purpose widgets to tabletops while providing the flexibility of on-screen controls. Madgets maintain consistency between physical controls and their digital state. BendDesk represents our vision of a multi-touch enabled office environment. Our pattern language captures knowledge for the design of interactive tabletops. For each project, we describe its technical background, present the current state of research, and discuss future work.
\end{abstract}

ACM Classification: H5.2 [Information interfaces and presentation]: User Interfaces. - Input devices and strategies.

General terms: Design, Human Factors

Keywords: Interactive tabletops, tangible user interfaces, haptic feedback, actuation, curved surface, applications.

\section{INTRODUCTION}

Interactive surfaces have received much interest from the research community in the last two decades, and first commercial products, such as Microsoft Surface, have been released. Representing an interactive piece of furniture rather than an explicit working device, interactive tabletops could be seamlessly integrated in our everyday environment in the near future. In office environments, digital tables offer a suitable setting for collaborative work while providing documents on demand. Furthermore, direct manipulation is an easy way to interact with graphical objects and gestures add further degrees of freedom for interaction.

However, interactive tabletops are still far away from being an accepted alternative for the conventional desktop computer, which involves keyboard and mouse. While finger input is intuitive, it lacks precision and the limited haptic feedback of a planar surface forces the user to focus on the input element she touches instead of the data she manipulates. Text input, the most frequent operation in desktop applications, is still an unsolved issue on tabletops. Conventional keyboards are highly optimized input devices but their use on tabletops

Permission to make digital or hard copies of all or part of this work for personal or classroom use is granted without fee provided that copies are not made or distributed for profit or commercial advantage and that copies bear this notice and the full citation on the first page. To copy otherwise, or republish, to post on servers or to redistribute to lists, requires prior specific permission and/or a fee.

UIST'10, October 3-6, 2010, New York, New York, USA.

Copyright 2010 ACM 978-1-4503-0271-5/10/10...\$10.00. is questionable, because they consume much real estate, have a static visual appearance, and require the user to change her input modality from direct touch to an external device [1]. However, typing on on-screen keyboards is error-prone as users cannot feel the keys. In general, the transfer of desktop applications to interactive tabletops is problematic and requires to rethink the applied input concepts while regarding the specific characteristics of a tabletop setup.

My research intends to enable the introduction of everyday tasks to interactive surfaces by exploiting the table's advantages while finding solutions for the issues that hinder a direct transfer from traditional desktop applications to tabletops. SLAP Widgets combine the benefits of tangible generalpurpose controls with the flexibility of on-screen widgets. Madgets maintain the consistency between these physical controls and their internal digital state. This allows to apply techniques known from desktop applications to tangible widgets, such as undo, save and load. BendDesk explores the vision of a fully multi-touch enabled office environment. It joins a horizontal and a vertical interactive surface and makes no use of keyboard and mouse anymore. Finally, we intend to capture the knowledge of tabletop design in an HCI design pattern language. These projects are presented in the following.

\section{TRANSLUCENT TANGIBLE WIDGETS ON TABLETOPS: SLAP WIDGETS}

On-screen widgets, such as buttons, sliders, and knobs, are the prevalent input controls for GUI applications. They imitate the affordances of their real world counterparts, can change their appearance dynamically and may be shown or
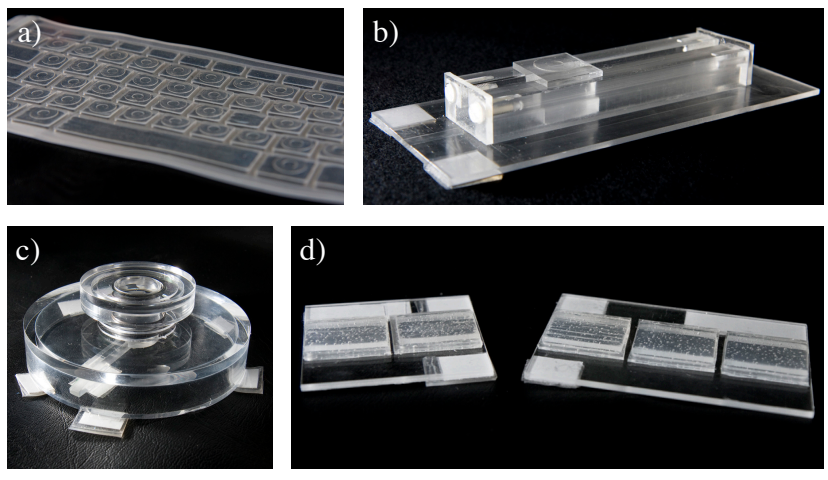

Figure 1: SLAP Widgets are translucent physical controls that use the table's back projection to change their visual appearance on the fly. a) Keyboard. b) Slider. c) Knob. d) Keypads. 
hidden on demand. However, their transfer to interactive tabletops is difficult. The intuitiveness of directly manipulating on-screen controls also involves a lower precision (fat finger problem), occlusion issues, and the need to focus widgets while controlling them. On the other hand, physical controls provide strong affordances and rich haptic feedback that guides the user's motion. They allow her to operate them in an eyes-free fashion while she focusses on the data she changes. However, physical controls are usually expensive, tethered, and static in terms of their visual appearance, which makes them unsuitable for the use on interactive tabletops.

Tangible user interfaces (TUIs) offer a solution to this issue. TUIs are physical objects that represent digital data and allow to manipulate the same [2]. In the context of interactive tabletops, several projects combine the haptic feedback of TUIs with a top or back projection to change their visuals dynamically [8]. However, most of these systems are either special purpose or offer only limited haptic feedback. We developed SLAP Widgets [11] that combine the benefits of physical controls and on-screen widgets.

SLAP Widgets are translucent general-purpose controls made of silicone and acrylic. Due to their physicality, they provide rich haptic feedback and allow an eyes-free interaction, and since they are translucent, we can use the back projection of our tabletop setup to change their visual appearance on the fly. Our current widget set includes buttons, sliders, keypads, and keyboards (Figure 1). We employ a visual tracking algorithm to sense fingers and objects on the tabletop. Each widget is mounted on a set of paper-based markers. The arrangement of these markers communicates the type, unique id, and state of each control to a camera beneath the table.

SLAP Widgets do not contain any electronics which makes them lightweight, low cost, and easy to build. We believe that this lowers the threshold for designers to build tabletop controls as no knowledge about electrical engineering is required. Furthermore, our widgets are ad-hoc tools that receive their specific purpose from the context they are used in. That is, a knob can be paired with an image on the table to change its brightness, or associated with a video to act as a jog wheel for fine-grain navigation. This tool character is particularly useful for text entry on tabletops, where collapsibility is an important requirement [1].

Our studies indicate that our physical controls can outperform on-screen controls in terms of task completion time and accuracy. Furthermore, users easily understood the concept of pairing physical widgets to on-screen objects. In future work, I want to conduct further studies on the performance of specific widgets. In particular, I intend to further iterate and evaluate the SLAP keyboard, as text entry on interactive tabletops is still an open problem.

\section{MAINTAINING CONSISTENCY: MADGETS}

Apart from all benefits, SLAP Widgets suffer from the inherent drawback that they only provide a unidirectional communication. When the user operates a physical control, e.g., when she moves a slider, the arrangement of markers changes. Our system then detects this change, updates the internal state of the widget, and renders the graphics accordingly.

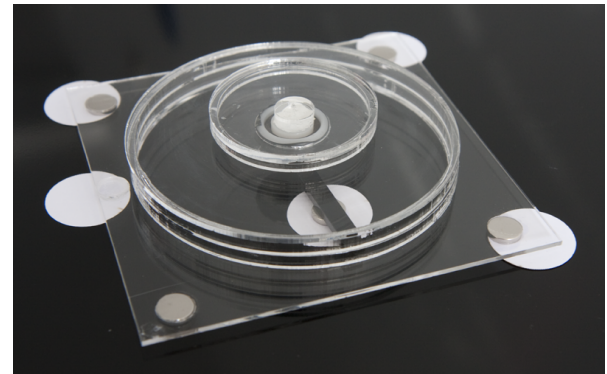

Figure 2: Madgets contain permanent magnets that allow their actuation on the tabletop by applying electromagnetic fields.

However, in the SLAP system, there is no way for the system to influence the physical configuration of a widget. If a slider is, e.g., paired with a video to control the playback volume, the position of the sliding knob obtrudes its physical state to the video object. It would be better though, if the slider was first set to the current volume after the pairing and then allowed the user to change that value. This is already a standard in many audio mixing desks that involve motorized sliders. Furthermore, SLAP Widgets can not reflect external events, e.g., when a widget's value is changed by a remote user. Instead the consistency between the physical and digital representation might break down, destroying the illusion that these merge into a single tangible user interface. Madgets [9] address this issue by providing a mechanism to actuate multi-element tangible widgets while keeping the advantages of lightweight and low-cost controls.

Similar to SLAP Widgets, Madgets (magnetic widgets) are general-purpose controls made from acrylic that transmit their type, id, and state to the system through an arrangement of markers. In addition, we attached permanent magnets to the controls (Figure 2) that allow our tabletop system to move, rotate, and configure our physical controls by applying dynamic electromagnetic fields.

Our tabletop system combines several technologies that integrate actuation, tracking, and visual output in a single device without the need for external cameras or projectors. The hardware setup is illustrated in Figure 3. Similar to the Actuated Workbench [4], we employ an array of electromagnets to move objects on the table. However, unlike previous approaches, we decompose all Madgets into multiple rigid bodies that are linked by joints. Each rigid body contains permanent magnets that are actuated individually. This allows the system, e.g., to move a Madget or to hold in place while moving subparts of it. To achieve the latter, we have to account for changing weight distributions during actuation.

An LCD panel that is backlit by an electroluminescent foil renders the graphical user interface that includes the dynamic visual appearance of each madget. For tracking, we intentionally discarded the use of external cameras to avoid occlusion issues. Instead, we applied a fiber optical tracking approach similar to FiberBoard [3] in combination with Diffused Surface Illumination (DSI). We placed fiber optics between the magnets and inside their cores, yielding a uniform grid which lets cameras beneath the table see past the actu- 
ation hardware. LEDs feed infrared light into an Endlighten layer. If a marker or finger is placed on the table, it reflects the infrared light down to the cameras. The markers of each Madget are circular and have an imprinted gradient that allows to determine their exact position even under the low resolution video. Furthermore, we can detect finger touches and basic gestures for moving and transforming objects.

Our actuation algorithm allows us to transfer many concepts from GUI applications to tangible widgets. Users can, e.g., undo/redo physical operations [4], save and load configurations, or interact with remote users via tangible interfaces [6]. Beyond that, the ability to hold an object in place while actuating subparts of it enables new actuation concepts. We developed, e.g., a physical radio button with three plates that can be raised and lowered using pulling or repelling magnetic fields. The Bell Madget actuates a small beater in vertical direction to ring a bell, providing mechanical audio feedback. Even more complex mechanisms are imaginable. We designed a lockable checkbox that can shift a bar beneath a button plate to block it, or - using the terminology of desktop applications - to "gray it out". Moreover, we can provide force feedback to the user. Finally, our setup allows us to transfer power to the Madgets. Our Induction Madget contains a coil that is connected to an LED. By applying an alternating electromagnetic field beneath the Madgets, we induce electrical power to the control. Using this technique, designers can, e.g., add sensors to the control without the need for batteries. The Gear Madget contains a pivot-mounted gear with attached permanent magnets and can act as a motor.

Although Madgets provide many degrees of freedom for actuation, their design is still easy. As SLAP Widgets, Madgets do neither contain batteries nor motors, which would cause a

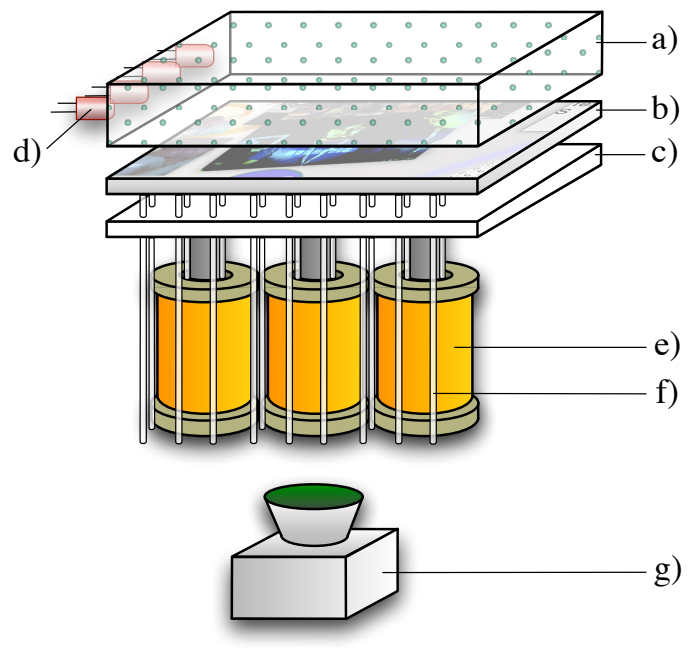

Figure 3: Hardware setup of our actuated interactive tabletop. Actuation: Electromagnets (e) generate magnetic fields to move and align Madgets or parts of them on the tabletop. Display: TFT panel (b) is backlit by electroluminescent foil (c). Tracking: LEDs (d) feed infrared light into an Endlighten acrylic (a). Objects placed on the table reflect IR light through fiber optical cables $(\mathrm{f})$ to IR cameras $(\mathrm{g})$ beneath the table. minimum weight and form factor. They are lightweight, lowcost, and can be prototyped rapidly. By implementing the actuation and tracking technology into the table and taking the electrical engineering away from the tangible controls, we believe that the simplicity of crafting Madgets might encourage industrial designers to build tabletop devices. Furthermore, the actuation based on magnetism allows to simulate physical effects. If a knob feels, e.g., too smooth-running the perceived friction can be increased by applying a larger pulling field to a permanent magnet in the turning arm.

After having implemented the system, we intend to develop a prototyping toolkit that allows to build and iterate Madgets. We will involve designers in the creation process and will conduct user studies to evaluate the usefulness and efficiency of the system. Moreover, we want to explore whether such a toolkit leads to more iteration cycles in medium fidelity prototypes as the perceived physical properties of a Madget can be refined without rebuilding the entire control.

A second aspect we want to investigate is the balance between the need for physical-digital consistency and the user's desire for control. While actuation helps to maintain the coherence between the physical controls and their internal digital state, it also deprives the user of control. This might become a crucial issue when an external event, e.g., in the context of remote collaboration, triggers an actuation that distracts the user or even interferes with her own tasks. We intend to find strategies and guidelines for situations where actuation consistency and user control conflict.

\section{AN INTERACTIVE DESK ENVIRONMENT: BENDDESK}

BendDesk [10] explores the vision of a fully multi-touch enabled office environment that does not use indirect input devices such as keyboard and mouse. It embodies a novel office desk environment where the entire surface is interactive while fitting into the user's ecology of the objects.

Inspired by the Sun Starfire project [7], BendDesk is an interactive tabletop that merges a vertical and horizontal interactive surface with a curve (Figure 4). A clear curved acrylic surface forms the base of the table. LED ribbons surrounding the acrylic feed infrared light into the surface. On top of the acrylic, we mounted a typical FTIR setup: a silicone layer and a diffusor. Three cameras behind the table track finger touches while short-throw projectors display the graphical user interface. An Anoto pattern imprinted on the diffusor can be used for high precision pen input. For both, display and rendering, we compensate for strong distortions that are induced by the curved shape of the table. When designing the system, we took special care that the user can sit in a comfortable position, reach the entire surface, and put everyday objects on the table.

After having constructed the system, we intend to evaluate the implications of this table design in two phases. In the first phase, we investigate basic direct manipulation gestures known from conventional interactive tabletops. In the second phase, we explore the application space that BendDesk enables. More precisely, we intend to find out to which extent such a system supports office tasks and which tasks still need a keyboard and mouse as input device. 


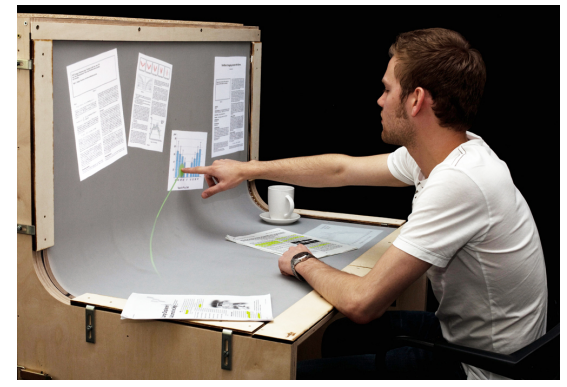

(a) BendDesk allows continuous finger gestures on a large interactive surface within the user's reach.

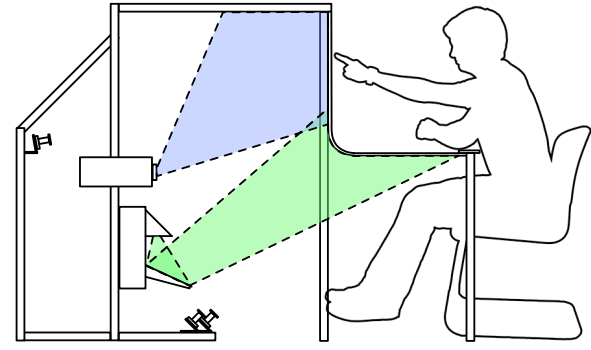

(b) Projectors and cameras behind the surface render the user interface and track finger input.

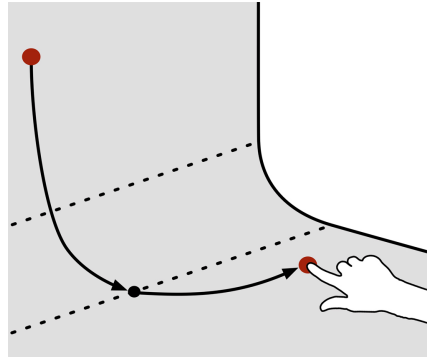

(c) When dragging across the curve, users tend to subdivide their trajectories into two convenient movements.

Figure 4: BendDesk table.

In our first studies, we investigated the performance of dragging on the different interactive areas of BendDesk. Our results show that dragging across the curve is significantly slower than on planar surfaces. Furthermore, we found out that dragging trajectories are significantly longer when approaching the curve in a flatter angle. Instead of dragging the direct line, users tend to minimize the dragging distance in the curve by subdividing the trajectory into two or more convenient movements (Figure 4c). Although BendDesk allows seamless dragging gestures across the entire surface, the curve is still perceived as a haptic barrier. In the future work of the project, we want to explore the properties of other gestures on our table, such as flinging or transforming objects using multiple fingers.

In the second phase of the project we want to explore potential applications for BendDesk. The different interactive areas can be used as logical workspaces when interacting with digital documents. Users could, e.g., create documents on the horizontal surface, store them in the curved area, and assemble them on the vertical surface into a "big picture". Another scenario is remote collaboration, where the horizontal surface acts as a private workspace, the curve as intermediate storage, and the vertical surface as a shared public space.

\section{CAPTURING KNOWLEDGE: DESIGN PATTERNS}

The progress in the field of interactive tabletops is still mostly driven by researchers. As part of my thesis, I intend to make the lesson learned from my research available to a broader audience, such as practitioners from other disciplines. In [8], we give an overview on tangible controls on tabletops with a focus on translucent general-purpose widgets. Furthermore, we are developing an $\mathrm{HCI}$ design pattern language for tabletops [5]. Our patterns provide solutions for recurring problems in the design of interactive tabletops. They follow a clear structure, and, since they are written in prose, they are comprehensible for researchers and practitioners across many disciplines. Our current language contains 22 patterns, e.g., for ergonomic considerations, interface design, or input techniques. In future, we will involve the community to further extend and refine the language.

\section{SUMMARY AND FUTURE WORK}

We presented projects that propose techniques to make interactive tabletops more compatible with the requirements of everyday tasks. In future work, we will further iterate and evaluate our systems. Moreover, we intend to implement real applications in order to find out to which extent interactive tables can be embedded into the flow of our everyday tasks.

\section{REFERENCES}

1. U. Hinrichs, M. Hancock, C. Collins, and S. Carpendale. Examination of text-entry methods for tabletop displays. Proc. TABLETOP '07, pp. 105-112. IEEE.

2. H. Ishii. Tangible bits: Beyond pixels. Proc. TEI '08, pp. $\mathrm{xv}-\mathrm{xxv}$. ACM.

3. D. Jackson, T. Bartindale, and P. Oliver. FiberBoard compact multi-touch display using channeled light. Proc. ITS '09, pp. 25-28. ACM.

4. G. Pangaro, D. Maynes-Aminzade, and H. Ishii. The actuated workbench: Computer-controlled actuation in tabletop tangible interfaces. Proc. UIST '02, pp. 181-190. ACM.

5. C. Remy, M. Weiss, M. Ziefle, and J. Borchers. A pattern language for interactive tabletops in collaborative workspaces. Proc. EuroPLoP '10. ACM. (to appear)

6. J. Richter, B. H. Thomas, M. Sugimoto, and M. Inami. Remote active tangible interactions. Proc. TEI '07, pp. 39-42. ACM.

7. B. Tognazzini. The "Starfire" video prototype project: A case history. Proc. CHI '94, pp. 99-105. ACM.

8. M. Weiss, J. D. Hollan, and J. Borchers. Augmenting interactive tabletops with translucent tangible controls. In Tabletops - Horizontal Interactive Displays, Human Computer Interaction Series, pp. 157-180. Springer Verlag.

9. M. Weiss, F. Schwarz, S. Jakubowski, and J. Borchers. Madgets: Actuating widgets on interactive tabletops. Proc. UIST '10. ACM. (to appear)

10. M. Weiss, S. Voelker, and J. Borchers. BendDesk: seamless integration of horizontal and vertical multi-touch surfaces in desk environments. Adjunct Proceedings ITS '09. ACM.

11. M. Weiss, J. Wagner, Y. Jansen, R. Jennings, R. Khoshabeh, J. D. Hollan, and J. Borchers. SLAP widgets: Bridging the gap between virtual and physical controls on tabletops. Proc. CHI '09. ACM. 